\title{
Oromucosal Capsule Dosage Form
}

National Cancer Institute

\section{Source}

National Cancer Institute. Oromucosal Capsule Dosage Form. NCI Thesaurus. Code C149732.

Solid single-dose preparation contained in a soft shell to be chewed or sucked to obtain a local effect in the oral cavity. 